$\mathrm{E}$

EVALUAR
2018, Vol. 18, No 1.

ISSN $1667-4545$

Recuperado de https://revistas.unc.edu.ar/index.php/revaluar

Laboratorio de Evaluación Psicológica y Educativa

Facultad de Psicología - Universidad Nacional de Córdoba

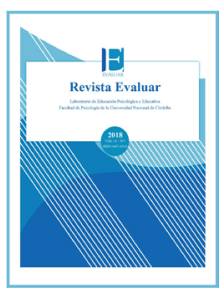

\title{
Propiedades psicométricas del Body Shape Questionnaire (BSQ-14) en mujeres universitarias mexicanas
}

\author{
Psychometric Properties of the Body Shape Questionnaire (BSQ-14) \\ in Mexican university Women
}

\author{
Karina Franco-Paredes * 1, M. Leticia Bautista-Díaz ${ }^{2}$, Felipe J. Díaz-Reséndiz ${ }^{1,3}$, \\ Elba M. Arredondo-Urtíz ${ }^{1}$ \\ 1 - Cuerpo Académico Comportamiento, Salud y Calidad de Vida, Centro de Investigación en Riesgos y Calidad de Vida. \\ Universidad de Guadalajara, México. \\ 2 - Cuerpo Académico Salud Emocional, Instituto de Ciencias de la Salud, Universidad Autónoma del Estado de Hidalgo, México. \\ 3 - Laboratorio de Análisis Conductual, Centro Universitario del Sur, Universidad de Guadalajara, México. \\ Introducción \\ Método \\ Resultados \\ Discusión \\ Referencias
}

Recibido: 03/10/2017 Revisado: 03/11/2017 Aceptado: 28/11/2017

\section{Resumen}

Contar con la versión breve de un cuestionario para evaluar la insatisfacción con la imagen corporal es importante tanto para la clínica como para la investigación, por ello, el objetivo de esta investigación fue analizar la estructura factorial, la validezconvergentey la consistenciainterna de una versión de 14 reactivos del Body Shape Questionnaire (Cooper, Taylor, Cooper, \& Fairburn, 1987) en una muestra de mujeres universitarias. Participaron400 universitariasmexicanas $(\mathrm{M}=19.43$ años, $\mathrm{DE}=1.11$ ), quienes respondieron el BSQ-14 y el Eating Attitudes Test (EAT-26). El análisis factorial confirmatorio comprobó la unidimensionalidad del BSQ-14 (CFI = .95; NFI $=.92 ; \mathrm{GFI}=.86 ; \mathrm{RMR}=.07 ; \mathrm{RMSEA}=.09)$. Asimismo, $\mathrm{el}$ BSQ-14 correlacionó positivamente con el EAT-26 $(r=.56$; $p<.001)$ y se encontró una excelente consistencia interna $(\alpha=.96 ;$ Omega $=.96)$. Se concluyó que la evidencia acerca de la validez y confiabilidad de las puntuaciones del BSQ-14 fue adecuada para mujeres universitarias mexicanas.

Palabras clave: estructura factorial, validez, consistencia interna, BSQ-14, mujeres

\begin{abstract}
A short version of a questionnaire to assess body dissatisfaction is important for both clinical and research application. Therefore, the aim of this study was to analyze the factorial structure, convergent validity and internal consistency of a 14-items short version of the Body Shape Questionnaire (Cooper, Taylor, Cooper, \& Fairburn, 1987) in a sample of university women. The sample included 400 Mexican university women $(\mathrm{M}=19.43$ years, $\mathrm{SD}=1.11)$, who completed the BSQ-14 and the Eating Attitudes Test (EAT-26). The confirmatory factor analysis corroborated the unidimensionality of BSQ-14 (CFI = .95; NFI = .92; GFI = .86; RMR = .07; RMSEA = .09). Also, BSQ-14 showed positive correlation with EAT-26 $(r=.56 ; p<.001)$ and an excellent internal consistency $(\alpha=.96$; Omega $=.96)$. It was concluded that the evidence about the validity and reliability of the BSQ-14 scores was adequate for university Mexican women.
\end{abstract}

Keywords: factorial structure, validity, internal consistency, BSQ-14, women

Cómo citar este artículo: Franco-Paredes, K., Bautista-Díaz, M. L., Díaz-Reséndiz, Arredondo-Urtiz, E. M.

*Correspondencia a: Karina Franco Paredes, Centro Universitario del Sur, Universidad de Guadalajara. Av. Enrique Arreola Silva 883, Colonia Centro, Ciudad Guzmán, C.P. 49000, Jalisco, México. karina.franco@academicos.udg.mx

Nota de los autores: Los autores agradecen a las asistentes de investigación Mayra G. Solórzano-Barragán, Jessica Reynoso-Sánchez, Karla S. GarcíaSolís y Guadalupe García-Palomera por el apoyo en la recolección de los datos. 


\section{Introducción}

La imagen corporal es una representación mental de la apariencia física (Raich, 2004; 2017; Thompson, Schaefer, \& Menzel, 2012; Smolak \& Thompson, 2001); es un constructo que incluye tres componentes: a) cognitivo; b) afectivo, y c) conductual. Estos componentes comprenden, a grandes rasgos, lo que se piensa, se valora $y$ se hace respecto a dicha representación (Baile-Ayensa, 2003; Raich, 2004; Stice, Maxfield, \& Wells, 2003; Thompson, Heinberg, Altabe, \& Tantleff-Dunn, 1999).

Las cogniciones, actitudes y conductas relacionadas con la imagen corporal están influidas, principalmente, por el contexto sociocultural, ya que la sociedad genera el estereotipo de ideal de belleza física a partir de la validación de pautas de forma y figura corporal que considera aceptables y/o deseables (Raich-Escursell, Sánchez-Carracedo, \& López-Guimera, 2008; Thompson et al., 2012). Así, vivir en una sociedad donde actualmente se equipara la delgadez con la belleza física, pone a algunas personas en una situación vulnerable que puede llevarlas a sentir insatisfacción con la imagen corporal, la cual se ha conceptualizado como un proceso que origina un grado elevado de malestar con el peso y la figura corporal alterando el curso natural de la vida cotidiana y que puede conducir a cogniciones, actitudes y conductas nocivas para la salud, no solo física sino también psicológica (National Eating Disorder Collaboration, 2017; Raich, 2017).

La insatisfacción con la imagen corporal es una característica definitoria de los trastornos de la conducta alimentaria (TCA; Bobadilla-Suárez \& López-Avila, 2014; Laporta-Herrero, JáureguiLobera, Barajas-Iglesias, \& Santed-Germán, 2016; Vaquero-Cristóbal, Alacid, Muyor, \& López-Miñarro, 2013); es un criterio diagnóstico central de la anorexia nerviosa (AN) y la bulimia nerviosa $(\mathrm{BN})$, y aunque no es criterio para el trastorno por atracón, también está presente en quienes lo padecen (DSM-5; American Psychiatric Association, 2013). Además, se ha sugerido que es un factor de riesgo y que incluso contribuye a la persistencia de estos trastornos (Stice, 2002) especialmente en mujeres, en quienes la prevalencia es mayor que en los varones (Gómez-Mármol, Sánchez-Alcaraz, \& Mahedero-Navarrete, 2013; Runfola et al., 2013; Vaquero-Cristóbal et al., 2013).

Por lo tanto, resulta necesario evaluar la insatisfacción con la imagen corporal como parte del conjunto de variables definitorias de los TCA. Los cuestionarios de autoinforme son los más utilizados para medir esta variable. Especialistas teórico-clínicos de los TCA diseñaron hace 30 años el Cuestionario de Imagen corporal (BSQ, por sus siglas en inglés; Cooper, Taylor, Cooper, \& Fairburn, 1987) ya que hasta ese momento los cuestionarios disponibles evaluaban el aspecto estético (atractivo) de la imagen y la única forma para medir la insatisfacción con la imagen corporal era la Eating Disorder Examination (EDE; Fairburn \& Cooper, 1993), una entrevista para evaluar los TCA en general pero no específicamente la insatisfacción con la imagen corporal.

El BSQ está conformado por 34 reactivos con seis opciones de respuesta, su objetivo es evaluar la insatisfacción con la imagen corporal, concretamente la debida al peso y forma del cuerpo. Las investigaciones realizadas en diversos países de dos continentes: Americano (CastrillónMoreno, Luna-Montaño, Avendaño-Prieto, \& Pérez-Acosta, 2007; Conti, Cordás, \& Dias de Oliveira-Latorre, 2009; Cooper et al., 1987; Da Silva, Ribeiro-Dias, Maroco, \& Alvares-DuarteBonini-Campos, 2014; Di Pietro \& da Silveira, 2009; Ghaderi \& Scott, 2004; Rosen, Jones, Ramírez, \& Waxman, 1996; Rousseau, Knotter, Barbe, Raich, \& Chabrol, 2005; Vázquez-Arévalo 
et al., 2011; Warren et al., 2008); y Europeo (Akdemir et al., 2012; Evans \& Dolan, 1993; Pook, Tuschen-Caffier, \& Brähler, 2008; RaichEscursell et al., 1996; Rousseau et al., 2005) han aportado evidencia acerca de la confiabilidad y la validez de las puntuaciones del instrumento, tanto en la versión original (34 reactivos) como en sus versiones cortas $(8,10,14$ y 16 reactivos).

Investigadores de Inglaterra y Estados Unidos propusieron y analizaron la confiabilidad y validez de ocho versiones breves diferentes del BSQ: 1) BSQ-16a, 2) BSQ-16b, 3) BSQ-8a, 4) BSQ-8b, 5) BSQ-8c, 6) BSQ-8c (Evans \& Dolan, 1993); 7) BSQ-14 (Dowson \& Henderson, 2001), y 8) BSQ-10 (Warren et al., 2008). En los estudios en los que se han analizado las propiedades psicométricas de las versiones cortas han participado poblaciones diversas, incluyendo tanto muestras clínicas como muestras generales de mujeres, varones y mujeres, preadolescentes, adolescentes y adultos. En todos los casos se ha encontrado una excelente consistencia interna con un rango Alfa $=.83-.97$ (Evans \& Dolan, 1993; Dowson \& Henderson, 2001; Ghaderi \& Scott, 2004; Kapstad, Nelson, Øverås \& Rø, 2015; Pook et al., 2008; Warren et al., 2008; Welch, Lagerstöm, \& Ghaderi, 2012) y estabilidad temporal (test-retest; $r=.90$ - .95; Ghaderi \& Scott, 2004; Welch et al., 2012). Como evidencia de validez se han demostrado diferencias grupales consistentes con la teoría (Dowson \& Henderson, 2001; Pook et al., 2008) y la correlación positiva con otros instrumentos ampliamente utilizados en el campo de los TCA (Dowson \& Henderson, 2001; Ghaderi \& Scott, 2004).

Las versiones cortas o breves de los cuestionarios cobran relevancia cuando se realiza investigación no solo sobre TCA, sino también en muestras generales, debido a que permiten evaluar diferentes variables para poder caracterizar, comparar o explicar los trastornos. En virtud de lo valioso que es contar con una versión breve del BSQ, que permita evaluar los mismos atributos que el cuestionario original, reduciendo los tiempos y la necesidad de recursos económicos y humanos, al momento de investigar, el propósito de este trabajo fue analizar la estructura factorial, la validez convergente y la consistencia interna del BSQ-14 en una muestra de universitarias mexicanas.

\section{Método \\ Participantes}

Se trabajó con una muestra de universitarias mexicanas $(\mathrm{n}=400)$ con un rango de edad de 18 a 22 años $(M=19.43$ años, $\mathrm{DE}=1.11)$ de una universidad pública ubicada en el sur del Estado de Jalisco, México. Para la selección de las participantes se utilizó un muestreo no probabilístico de tipo intencional. Considerando que se trabajaría con una muestra para el análisis factorial exploratorio (AFE) y con otra muestra para el análisis factorial confirmatorio (AFC) se estableció un tamaño muestral $n=200$ para cada análisis. Para establecer el tamaño de la muestra se utilizaron dos criterios: que tuviera más de 10 participantes por reactivo, por tratarse de una versión corta (Kim, 2005); y que incluyera al menos 200 casos, por tratarse de la evaluación de la calidad de un test (Ferrando \& Anguiano-Carrasco, 2010; Lloret-Segura, Ferreres-Traver, Hernández-Baeza, \& Tomás-Marco, 2014).

\section{Instrumentos}

Body Shape Questionnarie-14 (BSQ-14). Es la versión corta del cuestionario de autoinforme diseñado por Cooper et al. (1987) para medir la insatisfacción con la imagen corporal debida al 
peso y la forma del cuerpo. Consta de 14 reactivos directos con seis opciones de respuesta que van desde Nunca $=1$ hasta Siempre $=6$. Para la versión original de 34 reactivos Vázquez-Arévalo et al. (2011) hallaron evidencia de validez en una población de mujeres mexicanas, encontrando propiedades psicométricas muy satisfactorias: validez discriminante y predictiva $(\lambda$ de Wilks $=$ $\left..485, \chi_{(\mathrm{gl}=1)}^{2}=278.830, p<.001\right)$. Con el punto de corte de 110 el BSQ-34 clasificó correctamente a las participantes sin o con TCA y entre estas últimas distinguió a quienes presentaron $\mathrm{AN}$ o $\mathrm{BN}, \mathrm{y}$ la consistencia interna fue $\alpha=.98$.

Eating Attitudes Test-26 (EAT-26; Garner, Olmsted, Bohr, \& Garfinkel, 1982). Evalúa la presencia de sintomatología de TCA. Cuenta con 26 reactivos que se responden a través de una escala de seis puntos tipo Likert, deste Nunca $=1$ hata Siempre = 6; no obstante, se debe realizar una transformación de los datos de 0 a 3 para calcular la sumatoria, por lo que el rango de puntuación va de 0 a 78 puntos. Franco-Paredes, Solorzano, Díaz-Reséndiz e Hidalgo-Rassmussen (2016) encontraron que en mujeres mexicanas la consistencia interna del EAT-26 fue adecuada $(\alpha=.83) \mathrm{e}$ identificaron una estructura de tres factores.

\section{Procedimiento}

Se explicó el propósito de la investigación a las estudiantes en el salón donde tomaban clases y solo quienes aceptaron participar voluntariamente fueron conducidas a un aula del centro de cómputo de la universidad; el lugar contaba con iluminación y ventilación apropiadas para responder a los instrumentos en línea mediante la plataforma Lime Survey. En la primera pantalla las estudiantes leían una carta de consentimiento informado y si aceptaban participar procedían a contestar los instrumentos.
La presente investigación se realizó con estricto apego al Código Ético del Psicólogo (Sociedad Mexicana de Psicología, 2010) y a los principios éticos de la Asociación Americana de Psicología (2010) y es considerada de riesgo bajo según la Ley General de Salud de México (Gobierno de México, 2014).

\section{Análisis de datos}

El análisis de las propiedades psicométricas se realizó en dos etapas: 1. AFE y 2. AFC. En la primera etapa se trabajó con el paquete estadístico SPSS versión 17 (IBM Corporation, 2008). Se corroboró la posibilidad de factorizar de forma eficiente las variables originales mediante el método de Kaiser-Meyer-Olkin (KMO) y la prueba de esfericidad de Bartlett, comprobando la adecuación muestral de los datos y una correlación satisfactoria entre las variables analizadas. Se realizó un AFE con el método de extracción de máxima verosimilitud y rotación oblimin directa, siguiendo las recomendaciones de Lloret-Segura et al. (2014).

La unidimensionalidad del BSQ-14 se ratificó con el AFC realizado en el software estadístico AMOS versión 20. El modelo de un factor se evaluó considerando los siguientes índices de bondad de ajuste: como índice de parsimonia, el indicador $\chi^{2}$-normado $\left(\chi^{2} / g l\right)$; como índices de ajuste incremental, el índice de ajuste comparativo de Bentler (CFI); el índice de ajuste normado (NFI), y el índice de bondad de ajuste (GFI). Además, se consideraron los siguientes índices de ajuste absoluto: la raíz cuadrada media residual (RMR) y la raíz cuadrada media de error de aproximación (RMSEA). Para considerar que el modelo tiene un ajuste adecuado, el índice de parsimonia debe ser $<4$ (a menor índice, mejor ajuste); para CFI, NFI y GFI valores mayores o iguales a .95 indi- 
can buen ajuste y mayores o iguales a .90 indican un ajuste aceptable. Para RMR y RMSEA valores $<.05$ o a .08 son indicadores de un ajuste bueno o aceptable, respectivamente, a menor valor, mejor ajuste (Barret, 2007; Browne \& Cudeck, 1993; $\mathrm{Hu} \&$ Bentler, 1999). Finalmente, para analizar la consistencia interna del instrumento se calculó el coeficiente Alfa (en el software SPSS; IBM Corporation, 2008) y el coeficiente Omega, utilizando el software libre R (R Core Team, 2013).

\section{Resultados}

Homogeneidad de la prueba

Los resultados de la correlación reactivo-total mostraron que los valores de los coeficientes fueron mayores a .70 con un rango desde .71 para el reactivo 8 hasta .86 para los reactivos 2, 4 y 12 .

\section{Análisis factorial exploratorio}

El resultado del análisis de adecuación muestral $(\mathrm{KMO}=.96)$, al ser un valor que se encuentra cercano a la unidad, mostró una correlación alta (De Vicente y Oliva \& Manera-Bassa, 2003; Pérez, 2001); en tanto que la prueba de esfericidad de Barlett resultó significativa $(p<$ .0001 ), por lo que ambos indicadores confirmaron la pertinencia de realizar el análisis factorial (Pérez, 2001).

Se realizó un AFE con el método de extracción de máxima verosimilitud y rotación oblimin directa. El conjunto de reactivos quedó agrupado en un solo factor, con un valor propio superior a 1 y una varianza explicada de $68.17 \%$, con comunalidades (.53 a .78) y cargas factoriales (.73 a .88) altas para los 14 reactivos (ver Tabla 1 ).

Tabla 1

Cargas factoriales y comunalidades de los reactivos del BSQ-14

\section{Reactivo}

4. Estar desnuda (e.g. cuando te bañas) ¿te ha hecho sentir gorda?

12. Verte reflejada en un espejo o en un aparador ¿te ha hecho sentirte mal con tu figura?

2. Estar con chicas delgadas ¿te ha hecho fijarte en tu figura?

6. ¿Te has sentido excesivamente gorda o redondeada?

5. Comer dulces, pasteles u otros alimentos con muchas calorías ¿te ha hecho sentir gorda?

13. ¿Te has fijado más en tu figura estando en compañía de otras personas?

3. Al fijarte en la figura de otras chicas ¿la has comparado con la tuya desfavorablemente?

10. ¿Te has preocupado que otra gente vea llantitas alrededor de tu cintura o tu estómago?

1. ¿Te has preocupado tanto por tu figura que has pensado que tendrías que ponerte a dieta?

7. ¿Te has sentido acomplejada por tu cuerpo?

14. La preocupación por tu figura ¿te ha hecho pensar que deberías hacer ejercicio?

9. ¿Has pensado que la figura que tienes es debido a tu falta de control?

11. ¿Has pensado que no es justo que otras chicas sean más delgadas que tú?

8. Preocuparte por tu figura ¿te ha hecho ponerte a dieta?
Comunalidad Carga factorial

$.78 \quad .88$

.77

.88

.77

.87

.76

.72

Nota. $(n=200)$. 


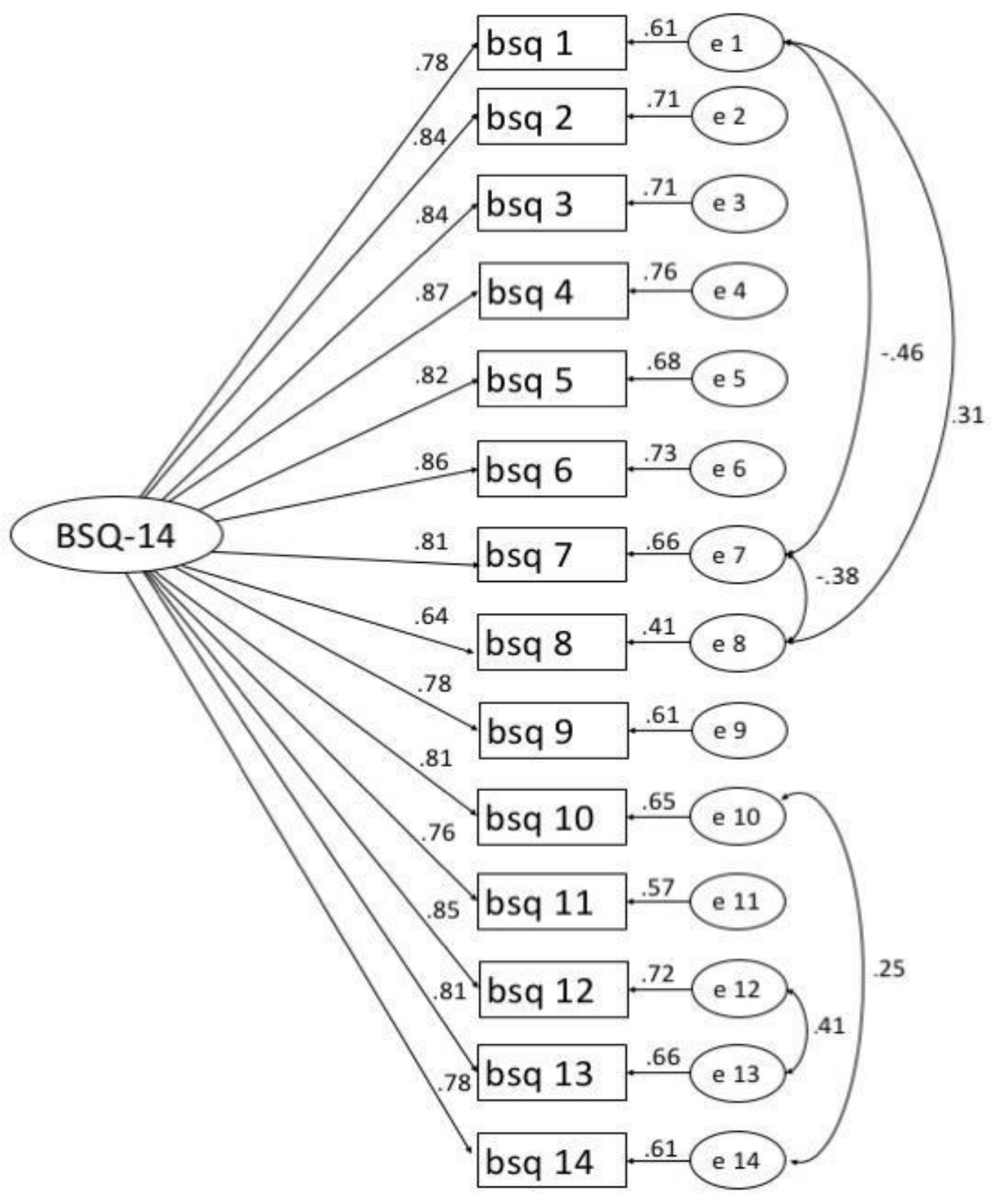

Análisis factorial confirmatorio

El AFC confirmó la unidimensionalidad del BSQ-14 (ver Figura 1). El modelo requirió concatenar cinco errores. En el modelo obtenido las saturaciones de los reactivos oscilaron entre .64 (bsq8) y .87 (bsq4). El índice de parsimonia para el modelo fue $\chi^{2} / g l=2.86$; los valores de los índices de ajuste incremental fueron $\mathrm{CFI}=.95, \mathrm{NFI}=.92$ y $\mathrm{GFI}=.86$ y los valores de los índices de ajuste absoluto fueron $\mathrm{RMR}=.07 \mathrm{y}$ RMSEA $=.09$.
Figura 1

Análisis factorial confirmatorio de la estructura del BSQ-14.

Nota. $(n=200)$.

\section{Validez convergente}

Se calculó la correlación entre el total del BSQ-14 y el total del EAT-26 y se obtuvo un coeficiente $r=.56(p<.001)$. 


\section{Consistencia interna}

El valor del coeficiente Alfa fue el mismo, tanto para la muestra total como para las submuestras utilizadas para el AFC y el AFE $(\alpha=$ .96). Asimismo, se calculó el coeficiente Omega, que para la muestra total fue Omega $=.97$ y para las submuestras fue Omega $=.96$.

\section{Discusión}

El propósito de esta investigación fue analizar la estructura factorial, la validez convergente y la consistencia interna del BSQ-14 en una muestra de universitarias mexicanas. Hasta donde se tiene conocimiento, es el primer estudio en México en el que se analiza una versión corta del BSQ después de la publicación del estudio en el que se analizaron las propiedades psicométricas de la versión original (BSQ-34) en estudiantes mexicanas (Vázquez-Arévalo et al., 2011). Los resultados del presente estudio coinciden parcialmente con los obtenidos en aquel estudio, por ejemplo, la consistencia interna es muy alta tanto para la versión original $(\alpha=.98)$ como para la versión corta $(\alpha=.97)$. Los resultados respecto a la dimensionalidad fueron diferentes debido a que para el BSQ-34 el AFE arrojó dos factores que agruparon 20 de los 34 reactivos y que explicaron $63.83 \%$ de la varianza, en tanto que para la versión BSQ-14 se demostró unidimensionalidad mediante AFE (varianza explicada $=68.17 \%$ ) y se ratificó con $\mathrm{AFC}$, modelo que obtuvo un ajuste general adecuado. Es decir que, en mujeres mexicanas, el BSQ-14 explicó una mayor cantidad de varianza con un solo factor en comparación con el BSQ-34. No obstante, durante el proceso de refinamiento estadístico del modelo fueron incluidas cinco correlaciones entre los errores asociados a los reactivos $1,7,8,10,12,13$ y 14 , lo que in- dica que el BSQ-14 tiene ítems redundantes con características similares para medir la insatisfacción con la imagen corporal; este es un resultado esperado en los modelos unidimensionales y es una característica constitutiva de las pruebas con esta estructura. Por este motivo se sugiere que futuras investigaciones aporten evidencia acerca de las propiedades psicométricas de las otras versiones cortas del BSQ (8 y 10 reactivos), con el fin de identificar la versión que presenta las mejores propiedades psicométricas.

En las investigaciones que han evaluado la dimensionalidad de las versiones cortas del BSQ en general se ha confirmado la estructura de una dimensión (Evans \& Dolan, 1993; Ghaderi \& Scott, 2004; Pook et al., 2008; Warren et al., 2008; Welch et al., 2012); sin embargo, en algunos casos el modelo obtuvo índices de ajuste pobres. Para el BSQ-14, Ghaderi y Scott (2004) confirmaron su unidimensionalidad tanto en muestra clínica como en estudiantes universitarias, con cargas factoriales mayores a .74, y 67\% de la varianza explicada, resultados que coinciden con los obtenidos en la presente investigación (cargas factoriales de .73 a .88 y varianza explicada del $68.17 \%$ ).

En general, la consistencia interna del BSQ (versión original y versiones cortas) se ha caracterizado por ser alta $(\alpha>.90)$. La consistencia interna para el BSQ-14 evaluada en investigaciones antecedentes ha fluctuado entre .93 y .96 (Dowson \& Henderson, 2001; Ghaderi \& Scott, 2004; Kapstad et al., 2015; Pook et al., 2008). La consistencia interna obtenida en este estudio también fue alta, calculando el coeficiente Alfa (.96) y el coeficiente Omega $(.96, .97)$. Hasta donde se tiene conocimiento este es el primer estudio en el que se analiza la consistencia interna del BSQ con el coeficiente Omega, a través del cual también se confirmó que es alta. Dunn, Baguley y Brunsden (2014), proponen que cuando se ha confirmado la estructura unidimensional de una escala es 
posible utilizar el coeficiente Omega, y que es mejor índice de consistencia interna que el Alfa de Cronbach, ya que minimiza el error asociado al considerar las respuestas de una escala Likert como variables cuantitativas continuas, por lo que establece supuestos más realistas, reduce la probabilidad de errores de estimación de la consistencia interna y refleja mejor tanto las estimaciones verdaderas como la variabilidad en el proceso de estimación. Así, se considera que el coeficiente Omega es de mejor ajuste a este tipo de estudios ya que permite una estimación más precisa de la confiabilidad del cuestionario al eliminar el efecto del número de reactivos y el número de opciones de respuesta que se generan al utilizar el coeficiente Alfa, además de tomar en cuenta las cargas factoriales (Schweizer, 2011).

En la presente investigación también se generó evidencia sobre la validez convergente de las puntuaciones del BSQ-14 debido a que correlacionaron positivamente con el EAT-26 $(r=.57)$, el cual es un instrumento ampliamente utilizado en el campo de los TCA. Este resultado es consistente con resultados de estudios precedentes, en los que se ha demostrado que en las mujeres universitarias la insatisfacción con la imagen corporal se asocia positivamente con la presencia de conductas y actitudes alimentarias anómalas ( $p$. ej., Dowson \& Henderson, 2001).

Una limitación del presente estudio fue que solo incluyó mujeres universitarias mexicanas, por lo que es necesario trabajar con mujeres de diferentes edades, dado que la presencia de insatisfacción con la imagen corporal también es alta en niñas, adolescentes y adultas, así como analizar si existe invarianza factorial entre las diferentes muestras. Asimismo, es importante considerar muestras clínicas para aportar mayor evidencia acerca del comportamiento del constructo en diferentes muestras.

Los hallazgos obtenidos permiten concluir que se confirmó la unidimensionalidad del instrumento y una excelente consistencia interna para evaluar la insatisfacción con la imagen corporal en mujeres universitarias mexicanas. No obstante, las altas cargas factoriales de los reactivos, las correlaciones de los errores en el modelo y la consistencia interna alta sugieren que es necesario analizar otras versiones cortas del instrumento.

\section{Referencias}

Akdemir, A., Inandi, T., Akbas, D., Kahilogullari, A. K., Eren, M., \& Canpolat, B. I. (2012). Validity and reliability of a Turkish version of the Body Shape Questionnaire among female high school students: Preliminary examination. European Eating Disorders Review, 20(1), 114-115. doi: 10.1002/erv.1106

American Psychiatric Association. (2013). Diagnostic and statistical manual of mental disorders, fifth edition ( $5^{\mathrm{a}}$ ed.). DSM-V. Arlington, VAWashington, DC.: American Psychiatric Association Publishing. Author.

American Psychological Association. (2010). Ethical Principles of Psychologists and Code of Conduct. Washington, DC: Author. Recuperado de https://www. apa.org/ethics/code/principles.pdf

Baile-Ayensa, J. I. (2003). ¿Qué es la imagen corporal? Revista de Humanidades Cuadernos del Marqués de San Adrián, 2, 1-17. Recuperado de http://www. unedtudela.es/revista_humanidades

Barrett, P. (2007). Structural equation modelling: Adjudging model fit. Personality and Individual Differences, 42(5), 815-824. doi: 10.1016/j.paid.2006.09.018

Bobadilla-Suárez, S., \& López-Avila, A. (2014). Distorsión de imagen corporal percibida e imaginada. Un posible factor para la obesidad y el sobrepeso en mexicanos. Revista Médica del Instituto Mexicano del Seguro Social, 52(4), 408-414. Recuperado de http:// revistamedica.imss.gob.mx/es

Browne, M. W., \& Cudeck, R. (1993). Alternative ways of 
assessing model fit. En K. A. Bollen \& J. S. Lonj (Eds.), Testing structural equation models (pp.136166). Newbury Park, CA: Sage.

Castrillón-Moreno, D., Luna-Montaño, I., Avendaño-Prieto, G. \& Perez-Acosta, A. M. (2007). Validación del Body Shape Questionnaire (Cuestionario de la Imagen Corporal, BSQ) para población colombiana. Acta Colombiana de Psicología, 10(1), 15-23. Recuperado de http://editorial.ucatolica. edu.co/ojsucatolica/revistas_ucatolica/index.php/ acta-colombiana-psicologia

Conti, M. A., Cordás T. A., \& Dias de Oliveira-Latorre, M. R. (2009). A study of the validity and reliability of the Brazilian version of the Body Shape Questionnaire (BSQ) among adolescents. Revista Brasileira de Sáude Materno Infantil, 9(3), 331-338. doi: 10.1590/ S1519-38292009000300012

Cooper, P. J., Taylor, M. J., Cooper, Z., \& Fairburn, C. G. (1987). The development and validation of the Body Shape Questionnaire. International Journal of Eating Disorders, 6(4), 485-495. doi: 10.1002/1098-108x(198707)6:4\%3C485::aid-eat2260060405\%3E3.0.co;2-o

Da Silva, W. R., Ribeiro-Dias, J. C., Maroco, J., \& Alvares-Duarte-Bonini-Campos, J. (2014). Confirmatory factor analysis of different versions of the Body Shape Questionnaire applied to Brazilian university students. Body Image, 11(4), 384-390. doi: 10.1016/j. bodyim.2014.06.001

De Vicente y Oliva, M. A., \& Manera-Bassa, J. (2003). El análisis factorial y por componentes principales. En J. P. Lévy-Mangin \& J. Varela-Mallou (Eds.), Análisis Multivariable para las Ciencias Sociales (pp. 327-360). Madrid: Pearson Educación.

Di Pietro, M., \& Da Silveira, D. X. (2009). Internal validity, dimensionality and performance of the Body Shape Questionnaire in a group of Brazilian college students. Revista Brasileira de Psiquiatría, 31(1), 2124. doi: 10.1590/S1516-44462008005000017

Dowson, J., \& Henderson, L. (2001). The validity of a short version of the Body Shape Questionnaire. Journal of
Psychiatric Research, 102(3), 263-271. doi: 10.1016/ S0165-1781(01)00254-2

Dunn, T. J., Baguley, T., \& Brunsden, V. (2014). From alpha to omega: A practical solution to the pervasive problem of internal consistency estimation. British Journal of Psychology, 105(3), 399-412. doi: 10.1111/ bjop. 12046

Evans, C., \& Dolan, B. (1993). Body Shape Questionnaire: Derivation of shortened "alternate forms". International Journal of Eating Disorders, 13(3), 315-321. doi: 10.1002/1098-108X(199304)13:3\%3C315::AID -EAT2260130310\%3E3.0.CO;2-3

Fairburn, C., \& Cooper, Z. (1993). The Eating Disorder Examination. En C. G. Fairburn \& G. T. Wilson. (Eds.), Binge eating: Nature, assessment and treatment (pp. 317-331). New York, NY: Guilford Press.

Ferrando, P. J., \& Anguiano-Carrasco, C. (2010). El análisis factorial como técnica de investigación en psicología. Papeles del Psicólogo, 31(1), 18-33. Recuperado de http://www.papelesdelpsicologo.es/

Franco-Paredes, K., Solorzano, M., Díaz-Reséndiz, F. J., \& Hidalgo-Rassmussen, C. (2016). Confiabilidad y estructura factorial del Test de Actitudes Alimentarias (EAT-26) en mujeres mexicanas. Revista Mexicana de Psicología, Suplemento, 278-279. ISSN 0185607-3

Garner, D. M., Olmsted, M. P., Bohr, Y., \& Garfinkel, P. E. (1982). The Eating Attitudes Test: Psychometric features and clinical correlates. Psychological Medicine, 12(4), 871-878. doi: 10.1017/S0033291700049163

Ghaderi, A., \& Scott, B. (2004). The reliability and validity of the Swedish version of the Body Shape Questionnaire. Scandinavian Journal of Psychology, 45(4), 319-324. doi: 10.1111/j.1467-9450.2004.00411.x

Gobierno de México. (2014). Ley General de Salud - México, Cámara de Diputados. Recuperada en Marzo 06, 2018, del sitio Web www.diputados.gob.mx/LeyesBiblio/regley/Reg_LGS_MIS.pdf http://www.diputados.gob.mx/LeyesBiblio/regley/Reg_LGS_MIS. pdf

Gómez-Mármol, A., Sánchez-Alcaraz, B. J., \& Mahede- 
ro-Navarrete, M. P. (2013). Insatisfacción y distorsión de la imagen corporal en adolescentes de doce a diecisiete años de edad. Ágora para la Educación Física y el Deporte, 15(1), 54-63. Recuperado de http://agora-revista.blogs.uva.es

Hu, L. T., \& Bentler, P. M. (1999). Cutoff criteria for fit indexes in covariance structure analysis: Conventional criteria versus new alternatives. Structural Equations Modeling, 6(1), 1-55. doi: 10.1080/10705519909540118

IBM Corporation. (2008). SPSS Statistics 17. [software de cómputo] Disponible en https://www-01.ibm.com/ software/mx/analytics/spss/products/statistics/

Kapstad, H., Nelson, M., Øverås, M., \& Rø, Ø. (2015). Validation of the Norwegian short version of the Body Shape Questionnaire (BSQ-14). Nordic Journal of Psychiatry, 69(7), 509-514. doi: 10.3109/08039488.2015.1009486

Kim, K. H. (2005). The relation among fit indexes, power, and sample size in structural equation modeling. Structural Equation Modeling, 12(3), 368-390. doi: $10.1207 /$ s 15328007 sem1203_2

Laporta-Herrero, I., Jáuregui-Lobera, I., Barajas-Iglesias, B., \& Santed-Germán, M. A. (2016). Body dissatisfaction in adolescents with eating disorders. Eating and Weight Disorders-Studies on Anorexia, Bulimia and Obesity. doi: 10.1007/s40519-016-0353-x

Lloret-Segura, S., Ferreres-Traver, A., Hernández-Baeza, A., \& Tomás-Marco, I. (2014). El análisis factorial exploratorio de los ítems: Una guía práctica, revisada y actualizada. Anales de Psicología, 30(3), 11511169. doi: 10.6018/analesps.30.3.199361

National Eating Disorders Collaboration (Febrero, 2017). What is body image? Recuperado de http://www.nedc.com.au/body-image

Pérez, C. (2001). Técnicas Estadísticas con SPSS. Madrid: Prentice-Hall.

Pook, M., Tuschen-Caffier, B., \& Brähler, E. (2008). Evaluation and comparison of different versions of the Body Shape Questionnaire. Psychiatry Research, 158(1), 67-73. doi: 10.1016/j.psychres.2006.08.002
R Core Team (2013). R: A language and environment for statistical computing. R Foundation for Statistical Computing, Vienna, Austria. Disponible en http:// www.R-project.org

Raich, R. M. (2004). Una perspectiva desde la psicología de la salud de la imagen corporal. Avances en Psicología Latinoamericana, 22(1), 15-27. Recuperado de http://revistas.urosario.edu.co/index.php/apl

Raich, R. M. (2017). La tiranía de la imagen. Barcelona: Singlatana.

Raich-Escursell, R. M., Mora, M., Soler, A., Avila, C., Clos, I., \& Zapater L. (1996). Adaptación de un instrumento de evaluación de la insatisfacción corporal. Clínica y Salud, 7(1), 51-66. Recuperado de http://clysa. elsevier.es/

Raich-Escursell, R. M., Sánchez-Carracedo, D., \& López-Guimera, G. (2008). Alimentación, modelo estético femenino y medios de comunicación: Cómo formar alumnos críticos en la educación secundaria. Barcelona: Graó.

Rosen, J. C., Jones, A., Ramirez, E., \& Waxman, S. (1996). Body Shape Questionnaire: Studies of validity and reliability. International Journal of Eating Disorders, 20(3), 315-319. doi: 10.1002/ (sici)1098-108x(199611)20:3\%3C315::aid-eat11\%3E3.3.co;2-n

Rousseau, A., Knotter, A., Barbe, P., Raich, R. M., \& Chabrol, H. (2005). Étude de validation de la version française du Body Shape Questionnaire. L'Encéphale, 31(2), 162-173. doi: 10.1016/ S0013-7006(05)82383-8

Runfola, C. D., Von Holle, A., Trace, S. E., Brownley, K. A., Hofmeier, S. M., Gagne, D. A., \& Bulik, C. M. (2013). Body dissatisfaction in women across the lifespan: Results of the UNC-SELF and gender and body image (GABI) studies. European Eating Disorders Review, 21(1), 52-59. doi: 10.1002/erv.2201

Schweizer, K. (2011). On the changing role of Cronbach's $\alpha$ in the evaluation of the quality of a measure. $E u$ ropean Journal of Psychological Assessment, 27(3), 143-144. doi: 10.1027/1015-5759/a000069 
Smolak, L. \& Thompson, K., (2001). Body image, eating disorders, and obesity in youth: Assessment, prevention, and treatment. Washington, DC: American Psychological Association.

Sociedad Mexicana de Psicología (2010). Código Ético del Psicólogo. ( $5^{\mathrm{a}}$ ed.) México: Trillas.

Stice, E. (2002) Risk a maintenance factor for eating pathology: A meta-analytic review. Psichologycal Bulletin, 128, 825-845. doi: 10.1037//0033-2909.128.5.825

Stice, E., Maxfield, J., \& Wells, T. (2003). Adverse effects of social pressure to be thin on young women: An experimental investigation of the effects of "fat talk". International Journal of Eating Disorders, 34(1), 108-117. doi: 10.1002/eat.10171

Thompson, J. K., Heinberg, L. J., Altabe, M., \& Tantleff-Dunn, S. (1999). Exacting beauty: Theory, assessment, and treatment of body image disturbance. Washington, DC: American Psychological Association. doi: 10.1037/10312-000

Thompson, J. K., Schaefer, L. M., \& Menzel, J. E. (2012). Internalization of thin-ideal and muscular-ideal. En T. F. Cash (Ed.), Encyclopedia of body image and human appearance (pp. 499-504). San Diego: Academic Press.

Vaquero-Cristóbal, R., Alacid, F., Muyor, J. M., \& López-Miñarro, P. A. (2013). Imagen corporal: revisión bibliográfica. Nutrición Hospitalaria, 28(1), $27-$ 35 Recuperado de http://www.nutricionhospitalaria. com/pdf/6016.pdf http://www.nutricionhospitalaria. com/pdf/6016.pdf

Vázquez-Arévalo, R., Galán-Julio, J., López-Aguilar, X., Álvarez-Rayón, G., Mancilla-Díaz, J. M., CaballeroRomo, A., \& Unikel-Santoncini, C. (2011). Validez del Body Shape Questionnaire (BSQ) en mujeres mexicanas. Revista Mexicana de Trastornos Alimentarios, 2(1), 42-52. Recuperado de http:// journals.iztacala.unam.mx/index.php/amta

Warren, C. S., Cepeda-Benito, A., Gleaves, D. H., Moreno, S., Rodriguez, S., Fernandez, M. C. ... Pearson, C. A. (2008). English and Spanish versions of the Body Shape Questionnaire: Measurement equivalence across ethnicity and clinical status. International Journal of Eating Disorders, 41(3), 265-272. doi: 10.1002/eat.20492

Welch, E., Lagerström, M., \& Ghaderi, A. (2012). Body Shape Questionnaire: Psychometric properties of the short version (BSQ-8C) and norms from the general Swedish population. Body Image, 9(4), 547-550. doi: https://doi.org/10.1016/j.bodyim.2012.04.009https:// doi.org/10.1016/j.bodyim.2012.04.009 